Revista de la Escuela de Ciencias de la Educación, año 12, nRo. 11, vol. 2, Julio a diciembre de 2016. Páginas 195-216. ISSN 1851-6297 - ISSN 2362-3349 (EN LINEA). FORMACIÓN PROFESIONAL Y COMPETENCIAS GENÉRICAS. MARIA CONCEPCIÓN BARRÓN TIRADO

\title{
FORMACIÓN PROFESIONAL Y COMPETENCIAS GENÉRICAS
}

\author{
María Concepción Barrón Tirado* \\ Universidad Nacional Autónoma de México. \\ baticon3@hotmail.com
}

Recibido: 06/03/2016 Aceptado: 05/06/2016

\begin{abstract}
Resumen
En este trabajo se identifican algunos rasgos distintivos de los modelos curriculares relacionados con la incorporación de las competencias genéricas en los planes de estudios en algunas instituciones de educación superior en México. Está divido en tres apartados; en el primero se presenta el panorama general en el que surge la educación basada en competencias como orientadora de la formación profesional ante los desafíos de los procesos de globalización económica, política y cultural; en el segundo apartado, se analizan las competencias genéricas y su incorporación en las algunas instituciones educativas; finalmente, en el tercer apartado se presentan algunas reflexiones generales a manera de cierre.
\end{abstract}

\section{Palabras clave:}

Competencias Genéricas - Competencias Básicas - Formación Profesional - Modelos Curriculares - Innovaciones Curriculares.

Licenciada, Maestra y Doctora en Pedagogía por la UNAM. Actualmente es Investigadora Titular "C" del Instituto de Investigaciones sobre la Universidad y la Educación (IISUE). Es miembro del Sistema Nacional de Investigadores, nivel II. Sus principales líneas de investigación son Didáctica, Currículum, Mercado de trabajo, Formación profesional y Estudios de posgrado. Ha desarrollado varias investigaciones individuales y colectivas entre las que destacan: "El impacto de los programas de evaluación en las universidades mexicanas", "Innovaciones curriculares y práctica docente", "Los modelos educativos y su impacto en los proyectos y prácticas curriculares", entre otras. Autora de los libros Formación profesional en la educación superior. Proyectos y prácticas curriculares y Universidades privadas. Profesionales de la educación. 
Revista de la Escuela de Ciencias de la Educación, año 12, nRo. 11, vol. 2, Julio a diciembre de 2016. Páginas 195-216. ISSN 1851-6297 - ISSN 2362-3349 (EN LÍNEA). FormACIÓN PROFESIONAL Y COMPETENCIAS GENÉRICAS. MARIA CONCEPCIÓN BARRÓN TIRADO

\begin{abstract}
In this article the author identifies some of the distinctive features of the curriculum models related to the inclusion of generic skills in the study programs of selected higher education institutions in Mexico. The study is divided into three parts. The first one presents a broad overview of the context in which competency-based education as a way to direct professional training in face of the challenges entailed by the economic, political and cultural globalization; the second part deals with the generic skills and their incorporation in some of the educational institutions; and in the third one the author presents some concluding reflections.
\end{abstract}

\title{
Keywords:
}

Generic Skills - Basic Skills - Professional Training - Curriculum Models Curriculum Innovation.

\section{Introducción}

Las problemáticas que enfrentan las sociedades contemporáneas, emanadas principalmente de los procesos de globalización, de la transferencia de tecnologías y de los nuevos requerimientos para la formación profesional, reclaman un soporte cultural distinto. Las fuerzas productivas tienen que estar altamente cualificadas, ser creativas y cada vez más autónomas; dichas condiciones se encuentran ligadas estrechamente a la educación (Castell, 1994).

En los modelos económicos tradicionales se asocia el crecimiento económico con el incremento de los factores de producción —incremento en la compra equipos y empleo de mayor número de trabajadores por parte de las empresas de un país - bajo la óptica de la llamada acumulación de capital. En las perspectivas económicas actuales se espera que la economía de un país crezca en función del aumento del valor económico generado por sus ciudadanos:

Los modelos económicos del "nuevo crecimiento" enfatizan la importancia del nuevo conocimiento, de la innovación y del desarrollo de capacidades humanas como fuentes de crecimiento económico sostenible. La educación y el desarrollo de capacidades humanas no solo permiten a los individuos agregar valor a la economía, sino contribuir al patrimonio cultural, participar en la sociedad, mejorar la salud de sus familias y comunidades, preservar el medio ambiente e incrementar su propia capacidad para continuar desarrollándose y realizando aportes; generando así un círculo virtuoso de realización personal y de contribuciones (UNESCO, 2008, p. 6).

Para el siglo XXI, la formación profesional de diversos países ha considerado el desarrollo de competencias que promuevan la creación de una mano 
Revista de la Escuela de Ciencias de la Educación, año 12, nRo. 11, vol. 2, JUlio a diciembre de 2016. Páginas 195-216. ISSN 1851-6297 - ISSN 2362-3349 (EN LINEAA). FoRMACIÓN PROFESIONAL Y COMPETENCIAS GENÉRICAS. MARIA CONCEPCIÓN BARRÓN TIRADO

de obra competitiva, para la cohesión social y para el desarrollo individual. La UNESCO (2008) reconoce que desde el ámbito económico se han señalado tres factores que conducen a un crecimiento basado en el desarrollo de la capacidad humana: una profundización en capital (capacidad de la mano de obra para utilizar un equipamiento más productivo que en sus versiones precedentes, alfabetización tecnológica); un trabajo de mejor calidad (mano de obra con más conocimientos, que puede añadir valor al resultado económico, a los problemas relacionados con el medio ambiente, a la seguridad alimentaria, a la salud y a la solución de conflictos); e innovación tecnológica (capacidad de la mano de obra para crear, distribuir, compartir y utilizar nuevos conocimientos).

Dicho enfoque, trae consigo implicaciones políticas que se orientan al aumento de la participación cívica, la creatividad cultural y la productividad económica, formando estudiantes, ciudadanos y trabajadores permanentemente dedicados a la tarea de crear conocimientos, innovar y participar en la sociedad del conocimiento, sacando provecho de esta tarea. Por lo tanto, se puede decir que las habilidades y las competencias que se promueven para del siglo XXI están más relacionadas con las necesidades de los modelos emergentes de desarrollo económico y social que con aquellas del siglo pasado al servicio del modo industrial de producción.

Al respecto, la OCDE planteó su postura a través de dos iniciativas: el Programa Internacional para la Evaluación de Estudiantes (PISA), lanzado en 1997 y la Definición y Selección de las Competencias (DeSeCo) de 2005. La DeSeCo tuvo como propósito definir un marco que guiara a largo plazo el desarrollo de evaluaciones de estas nuevas competencias, agrupando las competencias clave en tres grupos: a) uso interactivo de las herramientas; b) interacción entre grupos heterogéneos; c) actuar de forma autónoma. El eje de estos planteamientos gira en torno a la capacidad de los individuos para pensar por sí mismos y asumir la responsabilidad respecto de su aprendizaje y sus acciones (ITE, 2010).

Los resultados de DeSeCo constituyen los fundamentos teóricos de PISA; la finalidad de estas iniciativas fue explicar en qué medida los alumnos de la educación obligatoria han adquirido el conocimiento y las habilidades necesarias para participar activamente en la sociedad y para afrontar diversos desafíos en la vida futura. La incorporación de las habilidades y competencias genéricas y disciplinares en el currículo escolar, en los diversos niveles educativos, se dio paulatinamente y no de manera secuenciada, es decir, no se inició con los niveles básicos para llegar al superior, sino que, con base en las políticas educativas de cada país, se incluyeron de manera diferenciada.

En el caso de la educación superior, los nuevos contextos en los que se desarrolla se caracterizan por ser dinámicos y complejos. En un mundo globalizado, de alto nivel de desarrollo tecnológico, esta complejidad se puede 
Revista de la Escuela de Ciencias de la Educación, año 12, nRo. 11, vol. 2, Julio a diciembre de 2016. Páginas 195-216. ISSN 1851-6297 - ISSN 2362-3349 (EN LÍNEA). FormACIÓN PROFESIONAL Y COMPETENCIAS GENÉRICAS. MARIA CONCEPCIÓN BARRÓN TIRADO

traducir, entre otras cosas, en el surgimiento de nuevas áreas de conocimiento; cambios acelerados en la estructura del mercado laboral y de las profesiones; aceleración de la innovación científica y tecnológica; rapidez de los flujos de información; así como en la aceptación cada vez más generalizada de que tanto el conocimiento como la tecnología son los elementos de mayor impacto para el desarrollo de los países (Álvarez,1993).

Las instituciones de educación superior han asumido la responsabilidad de dar respuesta a los desafíos de la sociedad actual a través de la generación y la difusión de nuevos conocimientos en las áreas más diversas. Sin embargo, la calidad y eficacia con que éstas respondan a dichos retos dependerá, en buena medida, de las características que finalmente acaben adoptando los procesos de formación en sus planes y programas de pregrado, posgrado e investigación.

Por lo anterior, los retos que se presentan para la formación profesional conciernen tanto al ámbito disciplinario y científico, así como a una visión distinta de la realidad social y al conjunto de relaciones sociales en las que se inserta el profesionista. El conocimiento científico es concebido como un proceso enfocado a la invención de estrategias para el descubrimiento, la legitimación y la comunicación, en aras del desarrollo de la sociedad, por lo que se hace necesario reconocer que no sólo trata de un cambio científico y tecnológico, sino en un sentido más amplio implica un cambio cultural.

Como se puede ver, dos referentes básicos permean el sentido de la formación profesional: el campo de conocimiento y el ámbito del trabajo. Asimismo, la formación profesional comprende un conjunto de procesos sociales de preparación del sujeto con fines precisos para un posterior desempeño en el ámbito laboral que está basado en el abordaje, dominio y manejo de un cuerpo de conocimientos teóricos e instrumentales (saberes diferenciados) sobre determinado campo del saber, ciencia, quehacer o disciplina. Los conocimientos considerados fundamentales o básicos son esenciales para el desarrollo de las aptitudes individuales para el empleo; se busca un equilibrio entre éstos y las competencias metodológicas que permitan el aprender a aprender. Los conocimientos técnicos son aquéllos que permiten la identificación con una profesión y no se reducen únicamente a la adquisición de nuevos conocimientos demandados por sectores de vanguardia; dichos conocimientos permiten, además, el reconocimiento de aquellas competencias clave que han posibilitado a los profesionistas constituirse en gremios. Las aptitudes sociales atañen a las capacidades relacionales que tienen que ver con una gama de competencias requeridas en función del puesto de trabajo, autonomía, adaptación y creatividad, entre otras.

Es indispensable reconocer que la formación profesional no es tan sólo la suma de los roles que se le asignan en los distintos campos laborales, básicamente constituye un largo proceso en diversos planos: conceptual, reflexi- 
Revista de la Escuela de Ciencias de la Educación, año 12, nRo. 11, vol. 2, JUlio a diciembre de 2016. Páginas 195-216. ISSN 1851-6297 - ISSN 2362-3349 (EN LINEAA). FoRMACIÓN PROFESIONAL Y COMPETENCIAS GENÉRICAS. MARIA CONCEPCIÓN BARRÓN TIRADO

vo y práctico permeados por un compromiso ético con la sociedad y consigo mismo. La conciencia de las relaciones entre la persona, la naturaleza y la sociedad desborda la concepción del cambio tecnológico como simple acumulación de conocimientos, técnicas e instrumentos cada vez más complejos; por lo que hace falta una dinámica explícita y conscientemente innovadora en lo referente a la organización social y a los sistemas de valores, puesto que parece poco probable que resulte adecuado pensar simplemente en términos de adaptación de las maneras de vivir, de organizarse y de aprender que hoy en día son dominantes, sin revisar sus implicaciones éticas (INCANOP,1995).

Bajo esta perspectiva, en el contexto internacional existe un consenso en relación con la idea de la presencia de una desarticulación entre los modelos de formación profesional y los requerimientos productivos y tecnológicos. En general, las modificaciones en el currículo no van de la mano respecto de los requerimientos de conocimientos y de calificación; en las empresas las exigencias concretas de formación cambian más rápidamente que los planes de estudio de las IES. Sin embargo, esta situación es, en esencia, perfectamente lógica si se parte del hecho de que los tiempos, la dinámica y los ritmos del mundo de la economía y de las tecnologías difícilmente van a coincidir con los de la vida de las universidades: las exigencias de la producción no pueden ser las exigencias de la actividad académica.

Se reconoce la imposibilidad de adecuar, de manera generalizada, la oferta de educación con la oferta de trabajo, así como de considerar la relación entre educación y empleo como oferta y demanda dentro de un único mercado, cuando en realidad se está hablando de la articulación entre dos mercados autónomos: el de la educación y el de trabajo, cada uno de ellos con agentes específicos, instituciones y mecanismos propios de regulación entre oferta y demanda. (Planas, 2015). En los últimos años, la elevación del nivel de estudios de las generaciones no puede ser explicado solamente por razones económicas o por necesidades de la demanda de trabajo, difícilmente identificables a mediano y largo plazo, entre otras cosas, porque la expansión educativa está también relacionada con la función simbólica de la educación y de sus títulos. Depende de la multiplicidad de las funciones de la educación en los diversos sistemas sociales y de las políticas educativas de los Estados al pretender dar respuesta a las demandas políticas de la población y de las instituciones educativas.

\section{1.- Curriculum por competencias en México}

En México, la Secretaría de Educación Pública (SEP) estableció una política pública relacionada con las Instituciones de Educación Superior (IES), en particular con las universidades públicas, cuya columna vertebral lo constituye el Programa Integral de Fortalecimiento Institucional (PIFI), el cual contempla implícitamente un modelo educativo básico que debe servir de guía para 
Revista de la Escuela de Ciencias de la Educación, año 12, nRo. 11, vol. 2, Julio a diciembre de 2016. Páginas 195-216. ISSN 1851-6297 - ISSN 2362-3349 (EN LÍNEA). FormACIÓN PROFESIONAL Y COMPETENCIAS GENÉRICAS. MARIA CONCEPCIÓN BARRÓN TIRADO

el mejoramiento los programas educativos. Como consecuencia, las universidades públicas se han visto en la necesidad de elaborar formalmente sus modelos educativos y, por lo tanto, las instituciones de educación superior de carácter particular se encuentran en la misma situación, pues deben operar bajo la autorización de la SEP a través del denominado Reconocimiento de Validez Oficial de Estudios (RVOE). Aunado a lo anterior, en la última década del siglo $\mathrm{XX}$ y al inicio del presente siglo, emergieron organismos evaluadores y acreditadores de programas educativos preponderantemente, denominados Comités Interinstitucionales para la Evaluación de la Educación Superior (CIEES) y el Consejo para la Acreditación de la Educación Superior (COPAES). Como requisito para evaluar o acreditar sus programas educativos, las IES deben definir formalmente un modelo educativo.

Por otro lado, en las últimas décadas la Asociación Nacional de Universidades e Instituciones de Educación Superior (ANUIES) ha coadyuvado al Estado para el fortalecimiento y desarrollo de la educación superior. En su visión del sistema de educación superior al 2020 establece dos dimensiones referidas a los modelos educativos: innovación y formación integral. En la dimensión de innovación plantea la necesidad de diseñar modelos innovadores de aprendizaje y enseñanza, mientras que en la dimensión de formación integral se plantea la atención al alumno desde antes de su ingreso, asegurando su permanencia, desempeño, desarrollo y egreso. Entre los lineamientos estratégicos para el desarrollo de la educación superior, la ANUIES enfatiza la necesidad de:

- Reforzar las prácticas de gestión educativa y buscar una mayor eficiencia y eficacia de los procedimientos y reglas administrativas, del marco normativo y de la transparencia en el uso de los recursos.

- Innovar la formación de los estudiantes con estrategias, en donde la actualización de los programas educativos favorezca la adecuada correspondencia entre teoría y práctica; los problemas planteados se aborden de manera interdisciplinaria, y se promueva la flexibilidad curricular.

- Promover el desarrollo integral del estudiantado, mediante la incorporación de nuevos métodos que fomenten la formación permanente, el aprender a aprender, a emprender y a ser; el fomento de la creatividad y el espíritu de iniciativa; el desarrollo integral de las capacidades cognoscitivas y afectivas; el fomento del espíritu crítico y sentido de responsabilidad social.

- Aprovechar plenamente las tecnologías de la información y la comunicación. Desarrollar y consolidar los nuevos entornos pedagógicos en distintas modalidades, mediante la conformación de redes de educación abierta y a distancia, que articule los programas educativos.

- Formar y actualizar permanentemente al personal académico. 
Revista de la Escuela de Ciencias de la Educación, año 12, nRo. 11, vol. 2, JUlio a diciembre de 2016. Páginas 195-216. ISSN 1851-6297 - ISSN 2362-3349 (EN LINEAA). FoRMACIÓN PROFESIONAL Y COMPETENCIAS GENÉRICAS. MARIA CONCEPCIÓN BARRÓN TIRADO

- Redoblar esfuerzos para la transformación de la educación superior que contribuya a lograr pertinencia social y vinculación con el mundo laboral, así como a reforzar sus funciones deservicio a la sociedad, mediante el fortalecimiento y readecuación de las tareas de extensión educativa. (ANUIES, 2000, pp.216-217).

Ante este panorama, las reformas curriculares, sobre todo en la educación superior, han venido dando prioridad a fortalecer el vínculo entre educación y trabajo, a impulsar la educación continua y a distancia, a desarrollar programas de movilidad estudiantil y de cooperación interinstitucional haciendo énfasis en su carácter flexible y competitivo. Es así que, el mecanismo que orienta las reformas curriculares en las universidades mexicanas en la década pasada, se articula en torno a la flexibilidad curricular y al desarrollo de habilidades profesionales. Esto da sentido a reorientar la formación profesional hacia el currículo flexible diseñado por competencias (Díaz Barriga y Barrón, 2014).

Las diversas reformas realizadas en el sistema educativo mexicano en el siglo XXI responden, en cierta medida, a las dos propuestas más importantes que encabezan el modelo de formación: el Proyecto Tuning, impulsado por la Unión Europea (UE) y posteriormente el Proyecto Definición y Selección de Competencias (DeSeCo) (OCDE, 2005), que promueve la Organización para la Cooperación y el Desarrollo Económicos (OCDE) (Moreno, 2010). México, al ser miembro de la OCDE desde 1994, asume algunas de las políticas establecidas por este organismo y participa en el Programa Internacional para la Evaluación de Estudiantes o Informe PISA. También es importante mencionar el Proyecto América Latina Formación Académica, conocido como Alfa Tuning (Tuning América Latina, 2007), que deriva del Proyecto Tuning Europeo, y el cual busca la convergencia de estudios académicos con base en competencias y brinda posibilidades de articulación entre sistemas de educación superior. Dicho proyecto responde a los objetivos compartidos entre los países participantes con la finalidad de buscar una congruencia con las tendencias internacionales de la formación universitaria, en el marco de los principios de calidad y pertinencia.

Barrón (2000, p. 15) menciona que las tres razones que fundamentan el proyecto educativo basado en competencias en la visión de la Organización Internacional del Trabajo (OIT) son que: 1.-permite centralizar el crecimiento económico y el desarrollo social en el ser humano; 2.- posibilita crear mejores puestos de trabajo en donde la determinante es la capacidad de empleabilidad que tiene cada ser humano; y, 3.- se encuentra centrado en la necesidad de cambio.

La educación en competencias constituyó otro de los ejes del debate curricular de la década de los noventa en México; el antecedente fue el proyecto del Colegio Nacional de Educación Profesional Técnica (CONALEP) de 1993, 
Revista de la Escuela de Ciencias de la Educación, año 12, nRo. 11, vol. 2, Julio a diciembre de 2016. Páginas 195-216. ISSN 1851-6297 - ISSN 2362-3349 (EN LÍNEA). FormACIÓN PROFESIONAL Y COMPETENCIAS GENÉRICAS. MARIA CONCEPCIÓN BARRÓN TIRADO

el cual constituyó el proyecto piloto de educación técnica y modernización de la capacitación, financiado por el Banco Mundial e impulsado por las secretarías de Educación Pública (SEP) y del Trabajo y Previsión Social (STPS), y donde participaron representantes de trabajadores y empresarios. Más tarde, los modelos basados en las competencias se incorporaron en los modelos educativos de las instituciones de educación superior (1998-2000). La década de los noventa se caracterizó por la aparición de diversas propuestas de formación profesional que pretendieron estar acordes con las exigencias del mundo del trabajo, considerando las modificaciones sustanciales que han sufrido la estructura de las ocupaciones y de las profesiones.

En México no es posible hablar de un modelo curricular homogéneo para la educación superior, a diferencia de la educación básica, pues cada IES acuñó un marco conceptual en torno a las competencias y con base en este implantó su modelo educativo, en el marco de las políticas educativas nacionales e internacionales, por lo que es de llamar la atención la diversidad de acepciones que subyacen de manera explícita o implícita en el uso del término competencias.

Uno de los principales problemas a los que se han enfrentado los diseñadores del currículo en educación superior ha sido el de transitar de la lógica de las competencias técnico-laborales a la de competencias genéricas, académicas y sociofuncionales. En varios casos, la noción de competencia remite a un listado de conocimientos, habilidades y actitudes, de manera integral, pero en el momento de la interpretación y elaboración de programas concretos, se vuelve a privilegiar los primeros en detrimento de los dos últimos y no se logra en varias ocasiones establecer su articulación.

Empero, la educación basada en competencias en el ámbito educativo ha transitado por un sinnúmero de críticas y cuestionamientos de diversa índole. Desde el ámbito psicopedagógico se puede señalar la animadversión que se tiene al considerar el desarrollo de las competencias desde una perspectiva conductista, al reducir el aprendizaje de los estudiantes a resultados observables y cuantificables sin relación con los procesos mentales que subyacen a los comportamientos, ni con "el carácter polisémico de los significados, las intenciones, las disposiciones, así como la importancia decisiva de los contextos de actuación y de los aspectos éticos e interpersonales" (Pérez Gómez, 2008, p. 77). Asimismo, en el ámbito curricular, no pueden considerarse las competencias como un contenido añadido o prescriptivo, dejando de lado una visión integral de la formación del estudiante. Tampoco puede reducirse a un discurso normativo, que regule la vida académica de los estudiantes y docentes de manera acrítica en detrimento del desarrollo de la autonomía intelectual.

\section{2.- Competencias genéricas y planes de estudios}

En las diversas universidades públicas y privadas que acogieron este modelo es posible diferenciar el modelo académico del modelo curricular, el pri- 
Revista de la Escuela de Ciencias de la Educación, año 12, nRo. 11, vol. 2, Julio a diciembre de 2016. Páginas 195-216. ISSN 1851-6297 - ISSN 2362-3349 (EN LINEAA). FoRMACIÓN PROFESIONAL Y COMPETENCIAS GENÉRICAS. MARIA CONCEPCIÓN BARRÓN TIRADO

mero se liga a la visión y misión de la institución educativa y en el segundo se establecen los lineamientos generales del proyecto formativo de la institución.

Sobre lo que se ha escrito en torno a las competencias, en el Estado de Conocimiento sobre Curriculum, realizado por el Consejo Mexicano de Investigación Educativa (COMIE), se señaló que en las instituciones de educación superior:

Parte de los trabajos que abordan el diseño curricular por competencias lo enmarcan como un enfoque educativo centrado en el alumno, que propicia la participación activa del estudiante y que reconoce la variación en el ritmo y estilo de aprendizaje. Se le ubica como un enfoque que combina la formación teórica con la aplicación del conocimiento y su pretensión es lograr capacidades para realizar una determinada labor, tarea o actividad con un alto nivel de calidad y eficacia. En general, sus fundamentos se encuentran bajo la lógica de preparar una fuerza laboral más productiva y eficiente que dé respuesta a una economía globalizada (Díaz Barriga y Lugo 2003, p.71).

El diseño curricular por competencias ha planteado alternativas distintas a las tradicionales o bajo modalidades y componentes curriculares ya conocidos, pero con nuevas combinaciones y que ha llevado a la implementación de estrategias diversas para su diseño, implantación y evaluación. En este escenario, se observa que prácticamente la totalidad del sistema educativo mexicano ha vivido una efervescencia por las competencias.

A continuación, señalaremos algunos rasgos distintivos de los modelos curriculares en relación con la definición de competencias genéricas, así podremos identificar que ante la necesidad de superar el predominio de planes de estudio rígidos, organización académica rígida, normatividad obsoleta, concentración de la matrícula en carreras tradicionales, práctica docente tradicional, escasa vinculación entre dependencias y deficiente vinculación entre los programas académicos de docencia e investigación y los distintos sectores productivos, se generan propuestas alternativas con miras a superar dichos problemas.

Entre los rasgos que podemos mencionar, los modelos incluyen como componentes claves, entre otros, la flexibilidad curricular, las competencias genéricas o básicas, disciplinarias y profesionales, una visión interdisciplinaria de los problemas, la actualización permanente de los programas educativos, la incorporación de métodos activos centrados en el aprendizaje, la adopción del paradigma de la educación permanente como elemento fundamental de su filosofía educativa, el fomento del pensamiento crítico y creativo, el sentido de responsabilidad social y la promoción del desarrollo humano sustentable:

Desde parámetros constructivistas, la educación basada en competencias, extiende la necesidad de lograr en los estudiantes la transferencia de 
Revista de la Escuela de Ciencias de la Educación, año 12, nRo. 11, vol. 2, Julio a diciembre de 2016. Páginas 195-216. ISSN 1851-6297 - ISSN 2362-3349 (EN LÍNEA). FormACIÓN PROFESIONAL Y COMPETENCIAS GENÉRICAS. MARIA CONCEPCIÓN BARRÓN TIRADO

los conocimientos no sólo a contexto inmediatos, sino a la vida misma, a lo que viven los estudiantes aquí y ahora, y también a lo que tal vez necesiten para poder potenciar su vida futura (Díaz Barriga y Lugo 2003, p.72).

Asimismo, se puede señalar que existen diversas acepciones sobre las competencias genéricas, en particular se han concebido las competencias como transversales y transferibles a varias profesiones a diferencia de las competencias profesionales que están vinculadas a cada profesión.

\subsection{Competencias genéricas: rasgos particulares}

La sociedad de la información y del conocimiento exige a las instituciones de educación superior ofrecer a sus estudiantes herramientas para aprender a aprender a lo largo de toda la vida y desarrollar una competencia ética. El proceso formativo debe estar orientado a promover que los estudiantes tomen decisiones, se comuniquen eficazmente, argumenten con propiedad, trabajen en equipo y se desempeñen autónomamente en contextos laborales cada vez más dinámicos.

En el contexto de la educación superior, se denominan competencias claves o genéricas aquéllas desarrolladas por la mayoría de las carreras y están relacionadas con cualidades asociadas con la formación universitaria e incluyen una serie de habilidades cognitivas y metacognitivas, conocimientos instrumentales y actitudes, consideradas valiosas en la sociedad del conocimiento (Yániz y Villardón, 2006).

Las competencias genéricas se caracterizan por propiciar el aprendizaje autónomo de los estudiantes, la toma de decisiones de manera consciente y responsable, así como el reconocimiento de sus fortalezas y debilidades. Se pueden señalar como competencias genéricas el trabajar en equipo, el comunicarse con los demás, el gestionar información a través de las tecnologías de la información.

Además, el concepto de competencias genéricas está asociado al concepto de las cualidades humanas a partir de los planteamientos de Sen (1999), así como de Walker y Nixon (2007), en los que la educación se concibe como un periodo en el que los individuos aprenden a diseñar un proyecto personal, profesional y social. La función de la escuela es ayudar a los estudiantes a construir un proyecto de vida comprometido con su momento histórico. Dichos principios constituyen los fundamentos, junto con el libro Los siete saberes de Edgar Morin (1998), de las competencias claves propuestas por la OCDE (2005): competencias para utilizar interactivamente y de forma eficaz las herramientas e instrumentos de todo tipo que requiere la sociedad, para funcionar en grupos sociales cada vez más complejos y heterogéneos y para actuar de forma autónoma.

La incorporación de las competencias claves supone un cambio en las 
Revista de la Escuela de Ciencias de la Educación, año 12, nRo. 11, vol. 2, JUlio a diciembre de 2016. Páginas 195-216. ISSN 1851-6297 - ISSN 2362-3349 (EN LINEAA). FoRMACIÓN PROFESIONAL Y COMPETENCIAS GENÉRICAS. MARIA CONCEPCIÓN BARRÓN TIRADO

formas de enseñar, aprender y evaluar. No constituye solamente una modificación superficial. "Supone establecer el aprendizaje activo de conocimiento útil como el centro de la vida escolar, lo que significa una transformación radical de la forma de concebir el currículo, los procesos de enseñanza-aprendizaje y evaluación, la organización de los contextos escolares y la propia función docente" (Pérez Gómez, 2008, p. 86.)

Para la adquisición y desarrollo de estas competencias se requiere un proceso de enseñanza, de aprendizaje y de evaluación, bajo el acompañamiento de un tutor. Para transitar de un trabajo dependiente del tutor a la autonomía del estudiante, Prégent, Bernard y Kozanitis (2009, p. 44) reconocen cinco niveles en dicho proceso: en el nivel 1, el alumno moviliza la competencia con la ayuda y bajo la constante supervisión de un experto, reconoce situaciones en las que puede ser utilizada y es consciente de sus limitaciones; en el nivel 2, se moviliza la competencia con la ayuda y supervisión frecuente de un experto, las acciones deben ser revisadas para garantizar el cumplimiento; en el nivel 3, moviliza la competencia bajo la supervisión periódica de un experto, pero con ayuda en caso de nuevas situaciones, debe argumentar sus elecciones, pero es capaz de discernir los matices. En los niveles 4 y 5 , se moviliza la competencia sin ayuda y sin supervisión. Sus acciones sólo requieren la supervisión de un experto para garantizar su desarrollo. En el nivel 5 se moviliza la competencia sin ayuda y sin supervisión. El estudiante muestra su iniciativa en situaciones de riesgo y puede predecir el impacto de sus decisiones.

La incorporación de las competencias genéricas en el curriculum puede hacerse a través de tres modalidades: de manera paralela, diferenciada o de manera integrada. En lo que se refiere a la modalidad paralela se puede identificar un grupo de competencias genéricas para la institución educativa, se diseñan cursos específicos para su desarrollo. Con relación a la modalidad diferenciada, se diseñan las competencias genéricas en función del perfil profesional, en el marco de una institución educativa, se plantean cursos específicos para su desarrollo o son trabajadas en las asignaturas establecidas. Por último, la modalidad integrada permite articular las competencias genéricas con las específicas en escenarios reales (Villardón, 2015).

\subsection{Incorporación de las competencias genéricas. A manera de ejemplo}

En México algunas instituciones de educación superior integraron las competencias genéricas de manera específica a los planes de estudio y establecieron lineamientos generales para su operación; a continuación, presentaré algunos casos a manera de ejemplo.

En la Universidad Autónoma de Nuevo León (UANL), a partir de la integración del perfil del egresado, que contempla las competencias específicas de una profesión, se determinan a) las competencias genéricas a través de las 
Revista de la Escuela de Ciencias de la Educación, año 12, nRo. 11, vol. 2, Julio a diciembre de 2016. Páginas 195-216. ISSN 1851-6297 - ISSN 2362-3349 (EN LÍNEA). FormACIÓN PROFESIONAL Y COMPETENCIAS GENÉRICAS. MARIA CONCEPCIÓN BARRÓN TIRADO

competencias instrumentales que comprenden las capacidades, destrezas y habilidades que tienen una función instrumental en el ámbito profesional actual y pueden ser de naturaleza lingüística, metodológica, tecnológica o cognoscitiva, propias del perfil profesional necesario para la competitividad internacional y local; b) las competencias de interacción social, cuya finalidad es facilitar el proceso de desarrollo humano personal e interpersonal y c) las competencias integradoras, que se relacionan con el desarrollo de conocimientos, habilidades, destrezas, actitudes y valores que integran tanto a las competencias instrumentales como a las de interacción social, y que permiten al egresado una formación integral que lo haga competitivo, tanto a nivel local, como nacional e internacional (UANL, 2011).

En la UANL, el desarrollo de las competencias genéricas está ligado a las asignaturas del área de formación general universitaria (competencia comunicativa, aplicación de las tecnologías de información, apreciación a las artes, ambiente y sustentabilidad, contexto social de la profesión, ética, sociedad y profesión) y, posteriormente, deberán ser retomadas en las experiencias de aprendizaje, incluyendo evaluaciones, del resto de las asignaturas propias de los diferentes planes de estudio, de tal manera que sean incorporadas transversalmente (UANL, 2011).

En el caso de la Universidad Autónoma de Baja California (UABC), en el modelo curricular se señala que el desarrollo de las competencias básicas, disciplinarias y profesionales estará acorde con la formación de cada profesión. Por lo que cada plan de estudios integrará los lineamientos específicos determinados por la institución y seguirá la estructura curricular general que comprende tres etapas: la básica, la disciplinaria y la terminal. La incorporación de las competencias generales se realizará en el marco de cada plan de estudios ligadas a la profesión de que se trate (UABC, 2013). Por ejemplo, en la licenciatura en Derecho se determinaron las asignaturas en función de las competencias generales y específicas, se señala que las competencias genéricas se desarrollan a la par que las disciplinarias y profesionales y son evaluadas a partir de las evidencias planeadas en cada programa.

En la licenciatura en educación de la UABC, también se trabaja baja esta lógica y se hace referencia a la ausencia de una propuesta didáctica para su desarrollo

El enfoque por competencias no trae aparejado un modelo didáctico específico, por lo que depende del profesorado cómo interpretar el enfoque para poderlo instrumentar. De ahí que se considera necesario desarrollar y discutir modelos didácticos a través de los cuales se desarrolla el enfoque por competencias en la educación superior (Cordero y Nassar, 2013, p.32). 
Revista de la Escuela de Ciencias de la Educación, año 12, nRo. 11, vol. 2, Julio a diciembre de 2016. Páginas 195-216. ISSN 1851-6297 - ISSN 2362-3349 (EN LINNEA). FoRMACIÓN PROFESIONAL Y COMPETENCIAS GENÉRICAS. MARIA CONCEPCIÓN BARRÓN TIRADO

Es importante comentar que en la estructura general de los programas o syllabus se contempla un apartado que hace alusión a las competencias generales y particulares por asignatura y en la evaluación se menciona la elaboración de un producto integrador de las evidencias de desempeño logrado por el estudiante a través de la conformación de un portafolio de evidencias.

En la Universidad Autónoma de Yucatán (UADY) se señala desde el inicio que el perfil de egreso debe formularse en competencias que debe desarrollar el estudiante para la vida y su ejercicio profesional. Las competencias de egreso se componen, a su vez, por competencias genéricas, disciplinares y específicas...la Universidad ha establecido 22 competencias genéricas, por lo que cualquier Plan de estudios de la UADY debe integrarlas y asegurar su desarrollo, de manera transversal, en las diferentes asignaturas que lo conforman (UADY, 2012, pp. 24-25).

En la UADY, el establecimiento de las competencias disciplinares y de las competencias específicas está en función del estudio de los referentes disciplinares y profesionales. Su desarrollo requiere de varias asignaturas que integren las competencias genéricas y las disciplinares para que, en conjunto, se logre el perfil de egreso. A partir de las competencias de egreso se definen las competencias genéricas, disciplinares y específicas y en función de ellas se determinan los contenidos que se relacionan con cada una de ellas, posteriormente, se definen las asignaturas.

También se establece que la determinación de los contenidos orienta el desarrollo de las competencias y si se requiere de una o más asignaturas. Para definir el número de asignaturas, es necesario tener en cuenta los siguientes criterios: la(s) competencia(s) que se busca(n) favorecer; los contenidos imprescindibles en función de la(s) competencia(s) a desarrollar; el agrupamiento lógico de los contenidos; los saberes esenciales; el número de créditos (UADY, 2012). Asimismo, se define la acreditación como la validación que garantiza que la o el estudiante ha alcanzado las competencias de la asignatura en un nivel de dominio:

El nivel de dominio se define como los atributos o características que describen el grado en que la o el estudiante ha desarrollado determinada(s) competencia(s) de una asignatura. Para fines de promoción de una o un estudiante, el nivel de dominio se representa de manera cuantitativa y cualitativa y debe registrarse en un documento validado por la Institución. Los niveles de dominio los define la o el profesor, según la naturaleza de la asignatura en su planeación didáctica, ya que tiene que describir aquellos atributos o características que se espera del estudiantado en cada uno de los niveles de dominio (UADY,2012, p. 50).

La Universidad Iberoamericana (UIA), institución de carácter privado, 
Revista de la Escuela de Ciencias de la Educación, año 12, nRo. 11, vol. 2, Julio a diciembre de 2016. Páginas 195-216. ISSN 1851-6297 - ISSN 2362-3349 (EN LÍNEA). FormACIÓN PROFESIONAL Y COMPETENCIAS GENÉRICAS. MARIA CONCEPCIÓN BARRÓN TIRADO

denomina competencias genéricas cuando caracterizan el desempeño del egresado de cualquier licenciatura, mientras que las específicas definen el quehacer de una profesión determinada. El perfil de egreso esta descrito en términos de competencias, cada una de ellas se descompone en objetivos generales de una o más asignaturas del plan de estudios y cada una de ellas contribuye al logro de una o más competencias del perfil de egreso (UIA, 2012). En los planes de estudios se han definido seis competencias genéricas, además de las académicas, profesionales y de inspiración ignaciana y su promoción debe llevarse de manera transversal en el plan de estudios de cualquier licenciatura.

La estructura de todo plan de estudios abarca tres elementos: competencias genéricas y específicas que caracterizan a los egresados, dimensiones o aspectos de la formación y áreas curriculares en las que se agrupan las asignaturas. Para su desarrollo, las competencias se traducen en objetivos de tal manera que, al alcanzarse éstos, se logran las competencias. A lo largo del plan de estudios y a través de las diferentes asignaturas, lo estudiantes habrán de ir desarrollando las competencias genéricas propias de la Universidad y las específicas relacionadas con cada licenciatura.

Las dimensiones incluyen aspectos de la formación profesional, social e integral universitaria. Las áreas curriculares son las siguientes: básica, mayor y menor; de reflexión universitaria, de servicio social, de síntesis y evaluación. Con la finalidad de evaluar las competencias de manera integral, se crearon tres espacios curriculares, cuya intención es promover que el estudiante integre, aplique y evalúe la adquisición de competencias genéricas y profesionales.

También se ha elaborado una "Guía del docente para el desarrollo de competencias" (UIA, 2012) en donde se explicitan los criterios didácticos para su desarrollo, haciendo énfasis en diversas estrategias de enseñanza, aprendizaje y evaluación. Se recomienda integrar la evaluación al proceso de aprendizaje a través de diversas estrategias, sugieren una serie de técnicas e instrumentos para evaluar las competencias de manera integrada, tales como la elaboración de proyectos, la participación en debates, la redacción de ensayos, los portafolios de obras creativas, entrevistas y prácticas profesionales. Todos ellos deben ir acompañados de una guía de observación que describa lo que se espera de los estudiantes en cada aspecto específico del desempeño, dichas listas pueden ser listas de cotejo, escalas o rúbricas (UIA, 2012). Para su implantación y desarrollo, la definición de los perfiles constituyó el eje que orientó la planificación curricular y didáctica. 
Revista de la Escuela de Ciencias de la Educación, año 12, nRo. 11, vol. 2, Julio a diciembre de 2016. Páginas 195-216. ISSN 1851-6297 - ISSN 2362-3349 (EN LINEA). FORMACIÓN PROFESIONAL Y COMPETENCIAS GENÉRICAS. MARIA CONCEPCIÓN BARRÓN TIRADO

\section{3.- Reflexiones finales}

Las competencias genéricas se han incorporado como un componente de los currículos de las instituciones de educación superior en México, con la particularidad de que se plantean de manera general para todos los planes de estudios de una misma institución educativa, precisando su especificidad para cada profesión. Son abordadas a la par que las competencias disciplinarias y profesionales a lo largo de la formación de los estudiantes, por lo que no se enseñan y aprenden de manera disociada de los contenidos disciplinares.

En los diversos modelos curriculares se establecen algunos criterios generales para su enseñanza, aprendizaje y evaluación; no existen reportes concretos que den cuenta acerca del proceso didáctico seguido en la práctica por los docentes para su evaluación en el aula.

Las evaluaciones reportadas por algunas instituciones educativas se han realizado con la finalidad de dar cuenta acerca de la formación de los estudiantes en cuanto al desarrollo de las competencias genéricas y se han reportado casos específicos en algunas licenciaturas (ver tabla 1). 
Revista de la Escuela de Ciencias de la Educación, año 12, nRo. 11, vol. 2, Julio a diciembre de 2016. Páginas 195-216. ISSN 1851-6297 - ISSN 2362-3349 (EN LINEA). FormACIÓN PROFESIONAL Y COMPETENCIAS GENÉRICAS. MARIA CONCEPCIÓN BARRÓN TIRADO

Tabla 1. Evaluación de competencias Genéricas

\begin{tabular}{|c|c|c|}
\hline $\begin{array}{l}\text { Licen- } \\
\text { ciatura/ } \\
\text { Institu- } \\
\text { ción }\end{array}$ & $\begin{array}{l}\text { Propósito y } \\
\text { Metodología }\end{array}$ & Resultados \\
\hline $\begin{array}{l}\text { Socio- } \\
\text { log ía/ } \\
\text { UABC }\end{array}$ & $\begin{array}{l}\text { Estudio de Per- } \\
\text { cepción estu- } \\
\text { diantil. } \\
\text { Cuestionario } \\
\text { acerca de las } \\
\text { competencias } \\
\text { básicas y profe- } \\
\text { sionales. Escala } \\
\text { Likert. (Ortega y } \\
\text { Reyes, 2012) }\end{array}$ & $\begin{array}{l}\text { Las competencias básicas presentan una pro- } \\
\text { porción en el nivel muy alto que corresponde a } \\
\text { la disposición a relacionarse con diferentes per- } \\
\text { sonas en } 75.0 \% \text {, comunicar tus ideas oralmen- } \\
\text { te en diferentes contextos, trabajar en equipo } \\
\text { con responsabilidad y flexibilidad, preocuparse } \\
\text { por la calidad de tu trabajo todas con } 58.3 \% \text {, } \\
\text { comprender e interpretar la información conte- } \\
\text { nida en escrito, conocimientos generales de las } \\
\text { ciencias sociales y adaptarse a nuevas situa- } \\
\text { ciones con } 50 \% \text {; en el nivel alto se encuentra } \\
\text { el analizar y sinterizar la información, organizar } \\
\text { tu tiempo y afrontar tu trabajo habitual y realizar } \\
\text { tu evaluación con un } 58.3 \% \text { cada una, comu- } \\
\text { nicarte por escrito } 50.0 \% \text { y utilizar técnicas de } \\
\text { aprendizaje eficaces } 41.7 \% \text {; y por último como } \\
\text { significativas las de nivel medio utilizar técnicas } \\
\text { de aprendizaje eficaces } 41.7 \text {; mientras que el } \\
\text { resto de las competencias oscilan de } 8.3 \% \text { a } \\
25 \% \text {. }\end{array}$ \\
\hline $\begin{array}{l}\text { I n ge - } \\
\text { nierías } \\
\text { Quími- } \\
\text { ca, Me- } \\
\text { catróni- } \\
\text { ca e In- } \\
\text { dustrial } \\
\text { Univer- } \\
\text { s i d a d } \\
\text { Politéc- } \\
\text { nica }\end{array}$ & $\begin{array}{l}\text { "La formación } \\
\text { de competen- } \\
\text { cias genéricas y } \\
\text { específicas en } \\
\text { el nivel superior: } \\
\text { El caso de los } \\
\text { estudiantes de } \\
\text { ingeniería en } \\
\text { la Universidad } \\
\text { Politécnica de } \\
\text { Tlaxcala (UPT)" } \\
\text { Cuestionario } \\
\text { acerca de las } \\
\text { competencias } \\
\text { básicas y profe- } \\
\text { sionales. Escala } \\
\text { Likert (Cisneros, } \\
\text { 2015). }\end{array}$ & $\begin{array}{l}\text { Se consideraron egresados de los años } 2009 \text { a } \\
2013 \text {, en total fueron } 941 \text { ingenieros. Los indica- } \\
\text { dores que se encontraron en "muy bueno" son } \\
\text { capacidad para trabajar en equipo, pensamien- } \\
\text { to creativo, identificar y solucionar problemas, } \\
\text { aprendizaje continuo, búsqueda de información, } \\
\text { apego a las reglas, puntualidad, conocimientos } \\
\text { generales de la disciplina, conocimientos técni- } \\
\text { cos, habilidades para la comunicación escrita } \\
\text { y/o gráfica, capacidad analítica y lógica, asumir } \\
\text { responsabilidades en toma de decisiones, habi- } \\
\text { lidad para el manejo de paquetes computacio- } \\
\text { nales. Los indicadores que se concentraron en } \\
\text { "bueno" son habilidades de gestión, capacida- } \\
\text { des de alta dirección; en "regular" es dominio } \\
\text { de inglés }\end{array}$ \\
\hline
\end{tabular}


Demostrar la relación positiva entre las competencias genéricas y la empleabilidad laboral inicial. I.- Evaluación de estudiantes, II.-Evaluación de los profesores para cada uno de los estudiantes evaluando las competencias genéricas desarrolladas en el aula y extra aula. II- Evaluación de empleadores (González y Mendoza, 2011).
Para la Fase I sis de que a mayor grado de desarrollo de las competencias genéricas instrumentales, mayor empleabilidad inicial, la hipótesis que afirmaba: a mayor grado de desarrollo de las competencias genéricas de interacción social, mayor empleabilidad inicial, no se encontró apoyo para la misma; y por último, la hipótesis de mayor grado de desarrollo de las competencias genéricas integradoras, mayor empleabilidad inicial se encontró apoyo a la misma. Para Fase dos se encontró que los profesores contaban con formación en competencias. Con respecto a la Fase 3, los empleadores observaron que: Todos los estudiantes tienen gran capacidad para el manejo eficiente de herramientas de TIC. El $60 \%$ trabajan bien en equipo, son líderes y pueden trabajar en ambientes multidisciplinarios; y el $40 \%$ restante manifestó que no trabajaban en equipo o no tuvieron personal asignado a su cargo. El $90 \%$ se comunicaban en forma oral de forma fluida, redactando documentos en idioma propio y otra lengua(s) extranjera, mientras que el $10 \%$ mencionó que no fue necesario evaluar esta competencia. El $70 \%$ adquirían y administraban eficientemente su propio conocimiento, mientras que un $20 \%$ no compartía la misma opinión y solo un $10 \%$ destacó que fueron desarrollando poco a poco dicha competencia. El $80 \%$ se adaptaban fácilmente a situaciones cambiantes e inesperadas, mientras que un $10 \%$ manifestó que desarrolló en forma gradual su competencia y un $10 \%$ restante, destacó que en la empresa difícilmente ocurren situaciones de este tipo. El $90 \%$ de los estudiantes tienen la habilidad de manejar lenguaje matemático y lógico, y el $10 \%$ restante manifestó no requerir esa habilidad. 
Revista de la Escuela de Ciencias de la Educación, año 12, nRo. 11, vol. 2, Julio a diciembre de 2016. Páginas 195-216. ISSN 1851-6297 - ISSN 2362-3349 (EN LÍNEA). FormACIÓN PROFESIONAL Y COMPETENCIAS GENÉRICAS. MARIA CONCEPCIÓN BARRÓN TIRADO

Como puede advertirse, los estudios acerca de la evaluación de competencias genéricas se realizan al final del proceso de formación de los estudiantes; generalmente, se utiliza la encuesta, de corte descriptivo, como método de investigación que se caracteriza por recoger información puntual sobre una temática específica que, en este caso, es el desarrollo de las competencias genéricas. Se puede mencionar que se trata de una encuesta de opinión a partir de lo que los profesores, alumnos y empleadores, han percibido y experimentado respecto a éstas. Este tipo de estudios son válidos desde la perspectiva de Roe (2003), al reconocer una temporalidad en el proceso de formación a través de tres etapas: básica, inicial y profesional. En la etapa básica el estudiante debe desarrollar una serie de competencias fundamentales para el ejercicio profesional; las etapas inicial y avanzada responden estrictamente al desempeño profesional y son evaluadas a través de diversas metodologías como los estudios de trayectorias profesionales, de egresados y estrictamente de seguimiento de egresados.

El modelo curricular por competencias enfrenta grandes desafíos en su diseño y desarrollo, en lo que concierne al primero, la elección de incorporar las competencias genéricas de manera paralela, diferenciada o de manera integrada, orienta las prácticas de los profesores en el aula. Supone estrategias didácticas diferenciadas, para el proceso de enseñanza, aprendizaje y evaluación, incluyendo actividades prácticas donde se despliegue el desarrollo de las competencias en las diversas asignaturas, lo que implica un mayor tiempo de dedicación para la preparación de clases, así como para la retroalimentación y seguimiento. Sin dejar de soslayar, los debates conceptuales en torno al enfoque mismo de la educación basada en competencias y sin lugar a dudas la evaluación, que dé cuenta si el alumno ha logrado desarrollar la competencia o todavía requiere apoyos específicos para lograrlo. Asimismo, para llevar a cabo todo ello se tendría que contar con personal docente de tiempo completo en las instituciones de educación superior y la realidad dista mucho de ello, dado que el mayor porcentaje de docentes es contratado por horas clase.

Otro elemento a considerar es la heterogeneidad en el perfil de ingreso a la educación superior de los estudiantes y el número de alumnos por grupo con los que se trabaja en las universidades públicas en México, que oscila entre los 40 y 60 y que cada profesor atiende en promedio tres grupos. Por otra parte, se requiere de una reflexión más profunda acerca de la formación de los estudiantes y del desarrollo de sus capacidades reflexivas y críticas, más allá de las exigencias utilitaristas y pragmáticas del mercado de trabajo.

Finalmente, hasta el momento en México, no se han reportado experiencias concretas que hablen de manera precisa acerca del proceso de apropiación y de evaluación de las competencias genéricas en el aula, lo que pone de manifiesto su complejidad que se revela desde el momento de acuñar un currículo por competencias. 
Revista de la Escuela de Ciencias de la Educación, año 12, nRo. 11, vol. 2, julio a diciembre de 2016. Páginas 195-216. ISSN 1851-6297 - ISSN 2362-3349 (EN LINEA). FoRMACIÓN PROFESIONAL Y COMPETENCIAS GENÉRICAS. MARIA CONCEPCIÓN BARRÓN TIRADO

Por lo que valdría la pena indagar en el futuro a partir de las siguientes interrogantes:

- ¿Cuáles son las claves del éxito para la adquisición de las competencias genéricas?

- ¿Qué formación deben tener los docentes para desarrollarlas a la par de los contenidos disciplinares o deberá de integrarse de otra manera el currículum?

- ¿Qué tipos de evaluación son apropiados para el seguimiento y la evaluación de las competencias genéricas?

- ¿Cómo no caer en visiones reduccionistas de los modelos de educación basados en el enfoque de competencias?

\section{Referencias Bibliográficas}

- Álvarez, J. M. (1993). El alumnado. La evaluación como actividad crítica de aprendizaje, Cuadernos de Pedagogía, (219), noviembre, pp. 28-32.

- Ananiadou, K. and Claro, M. (2009), "21st Century Skills and Competences for New Millennium Learners in OECD Countries". OECD Education Working Papers, (41), OECD Publishing. http://dx.doi.org/10.1787/218525261154

- Asociación Nacional de Universidades e Instituciones de Educación Superior (2000). La educación superior en el siglo XXI. México: ANUIES.

- Barrón, C (2015). Modelos curriculares y competencias genéricas. Algunas experiencias en la Educación Superior en México. Ponencia presentada en el II Encuentro Internacional Universitario 2015, "Las competencias genéricas en la educación superior", Pontificia Universidad Católica del Perú.

- Barrón, C. (2000). La educación basada en competencias en el marco de los procesos de globalización. En Valle, M. A. (coord.) Formación en competencias y certificación profesional, col. Pensamiento universitario, núm. 91 México: CESU-UNAM, pp. 17-44

- Barrón, C. y Marisa Ysunza M. (2003). Currículum y formación profesional. En Díaz Barriga, A. (coord.). La investigación curricular en México: la década de los noventa. México: COMIE. 125-163.

- Beneitone, P. et al. (2007). Reflexiones y perspectivas de la educación superior en América Latina. Bilbao, España: Universidad de Deusto.

- Castell, M. (1994). Flujos y redes e identidades: una teoría crítica de la sociedad informacional. En Castelll. M. et al. Nuevas perspectivas críticas en educación. Barcelona: Paidós. pp.15-53.

- Cisneros, F. (2015). La formación de competencias genéricas y específicas en el nivel superior. El caso de los egresados titulados de ingeniería Química, Mecatrónica e Industrial en la Universidad Politécnica de Tlaxcala (UPT). Tesis de doctorado en Educación, México, Universidad Autónoma de Tlaxcala. 
Revista de la Escuela de Ciencias de la Educación, año 12, nRo. 11, vol. 2, Julio a diciembre de 2016. Páginas 195-216. ISSN 1851-6297 - ISSN 2362-3349 (EN LÍNEA). FormACIÓN PROFESIONAL Y COMPETENCIAS GENÉRICAS. MARIA CONCEPCIÓN BARRÓN TIRADO

- Cordero, G. y Nassar, Y. (2013). "Modelo didáctico para la aplicación del enfoque por competencias en la formación de licenciados en ciencias de la educación". Revista Digital de Investigación Educativa Conect@2 VIII Edición. Diciembre Año III, (201).

- Corominas, E., Tesouro, M., Capell, D., Teixidó, J., Pèlach, J. y Cortada, R. (2006). "Percepciones del profesorado ante la incorporación de las competencias genéricas en la formación universitaria". Revista de Educación, 341, 301-336. Disponible en: http://dugi-doc.udg.edu/bitstream/handle/10256/9861/PercepcionesProfesorado.pdf?sequence=1

- Díaz Barriga, F. y Barrón, C. (2014). "Curricular changes in higher education in Mexico (2002-2012)" Journal of Modern Education Review, Academic Star Publishing Company, July, 28 ${ }^{\text {th }}, 2014$. http://www.sciedu.ca/journal/index.php/jct/article/ view/4865/3078

- Díaz Barriga, F. y Lugo, E. (2003). Desarrollo del currículo. En Díaz Barriga, A. (coord.). La investigación curricular en México: la década de los noventa. México: COMIE, pp. 63-125.

- EURIDYCE (2002). Las competencias clave. Un concepto en expansión dentro de la educación general obligatoria. EURIDYCE. La Red Europea de Información en Educación, p. 2. Recuperado de: http://eurydice.org.

- González, V. y Mendoza, J. (2011). La influencia de las competencias genéricas en la empleabilidad laboral inicial. Caso de estudio en la UANL. En InnOvaciOnes de NegOciOs 8(16): 391-413 (ISSN 2007-1191).

- Institut Catalá de Noves Professions (INCANOP) (1995). Nuevas Tecnologías, nuevas profesiones. Las nuevas tecnologías y los efectos que ejercen sobre las profesiones y la formación en sectores promisorios de la actividad económica, en Cataluña y en Europa. México: ANUIES-Institut Catalá de Noves Professions.

- Instituto de Tecnologías Educativas (ITE) (2010). Habilidades y competencias del siglo XXI para los aprendices del milenio en los países de la OCDE. Ministerio de Educación. España. Disponible en: http://recursostic.educacion.es/blogs/europa/ media/blogs/europa/informes/Habilidades_y_competencias_siglo21_OCDE.pdf

- Lacavex, M. A., Villa, R., Flores, E. Vicente, A. (2007). "El nuevo plan de estudios por competencias de la licenciatura en Derecho de la Universidad Autónoma de Baja California”. En Academia. Revista sobre enseñanza del Derecho. Año 5, (10), pp. 211-259.

- Lévy- Levoyer, C. (1997). Gestión de las competencias. Barcelona: Ediciones Gestión 2000.

- Moreno, T. (2010). El currículum por competencias en la universidad: más ruido que nueces. Revista de la Educación Superior, Vol. XXXIX (2), No. 154, abril-junio, pp. 77-90.

- Morin, E. (1998). Los sietes saberes necesarios para la educación del futuro. México, UNESCO, Correo de la UNESCO. 
Revista de la Escuela de Ciencias de la Educación, año 12, nRo. 11, vol. 2, Julio a diciembre de 2016. Páginas 195-216. ISSN 1851-6297 - ISSN 2362-3349 (EN LINEA). FORMACIÓN PROFESIONAL Y COMPETENCIAS GENÉRICAS. MARIA CONCEPCIÓN BARRÓN TIRADO

- OCDE (2005). La Definición y Selección de Competencias Clave (DeSeCo). Recuperado de: http://www.deseco.admin.ch/bfs/deseco/en/index/03/02.parsys.78532. downloadList.94248.DownloadFile.tmp/2005.dscexecutivesummary.sp.pdf [fecha de consulta: septiembre de 2015].

- Ortega, L. y Reyes, O. (2012). ¿Cómo formar competencias profesionales en el nivel superior?. En Revista Electrónica Educare Vol. 16, № 2, [25-54], mayo-agosto, 2012, ISSN: 1409-42-58, URL: http://www.una.ac.cr/educare.

- Pérez Gómez, A (2008). ¿Competencias o pensamiento práctico? La construcción de los significados de representación y de acción. En Gimeno Sacristán, J. (Comp.), Educar por competencias ¿qué hay de nuevo?, Madrid: Morata. p.p.59102.

- Perrenoud, P. (2004). Diez nuevas competencias para enseñar. Barcelona: Graó.

- Perrenoud, P. (2007). Desarrollar la práctica reflexiva en el oficio de enseñar. Profesionalización y razón pedagógica. México: Colofón.

- Planas, J. (2015). "Adecuar la oferta de educación a la demanda de trabajo. ¿Es posible?" Conferencia XIII Congreso Nacional de Investigación Educativa. Consejo Mexicano de Investigación Educativa (COMIE), Chihuahua, 16-20 noviembre de 2015

- Prégent, R., A. Bernard. H. Kozanitis, A (2009). Enseigner à l' université dans une approche-programme. Canada, Presses Internationales Polytechnique.

- Roe, R. (2003), ¿Qué hace competente a un psicólogo?. En Papeles del Psicólogo, Revista del Colegio Oficial de Psicólogos, (83), diciembre.

- Secretaría de Educación Pública, México. (2005). Modelo Educativo Basado en Competencias del subsistema de Universidades Politécnicas. México, Distrito Federal: CUP.

- Sen, A. (1999). Development as Freedon. Nueva York: Knopf.

- Tuning-América Latina (2007). Reflexiones y perspectivas de la Educación Superior en América Latina. Informe final. España: Publicaciones de la Universidad de Deusto.

- UNESCO (1998). La educación superior en el siglo XXI. Visión y acción. Debate temático: Las exigencias del mundo del trabajo. Conferencia Mundial sobre la Educación Superior. París, 1-8 de octubre, París: UNESCO.

- UNESCO (2008) Estándares de competencias en tic para docentes. Disponible en http://www.oei.es/tic/UNESCOEstandaresDocentes.pdf

- Universidad Autónoma de Baja California (UABC) (2013). Modelo educativo. Recuperado de: http://www.uabc.mx/formacionbasica/modeloedu.htm

- Universidad Autónoma de Campeche (UACAM) (2012). Modelo educativo. Recuperado de: http://uacam.mx/?modulo=paginas\&acciones=ver\&id_pagina=ekVd

- Universidad Autónoma de Chihuahua (UACH) (2008). Modelo educativo. Recuperado de: http://www.uach.mx/academica_y_escolar/modelo_educativo/2008/05/21/ 
Revista de la Escuela de Ciencias de la Educación, año 12, nRo. 11, vol. 2, JUlio a diciembre de 2016. Páginas 195-216. ISSN 1851-6297 - ISSN 2362-3349 (EN LINNEA). FoRMACIÓN PROFESIONAL Y COMPETENCIAS GENÉRICAS. MARÍA CONCEPCIÓN BARRÓN TIRADO

modelo_educativo_uach/

- Universidad Autónoma de Nuevo León (UANL) (2008). Modelo académico de la UANL. México: UANL.

- Universidad Autónoma de Nuevo León (UANL) (2011). Modelo académico de la Universidad Autónoma de Nuevo León. México: UANL.

- Universidad Autónoma de Yucatán (UADY) (2002). Modelo educativo y académico. Recuperado de: http://www.uady.mx/pdfs/me.pdf.

- Universidad Autónoma de Yucatán (UADY) (2012). Modelo educativo para la formación integral. Recuperado de: http://www.dgda.uady.mx/media/docs/mefi_dgda. pdf

- Universidad Autónoma del Estado de Hidalgo (UAEH) (2006). Modelo educativo. Recuperado de: http://www.uaeh.edu.mx/docencia/docs/modelo_educativo_ UAEH.pdf

- Universidad Iberoamericana (UIA) (2012). Guía del docente para el desarrollo de competencias. México. UIA. Recuperado de http://prepajocotepec.sems.udg.mx/ sites/default/files/Enlaces/guia_docente_desarrollo_competencias.pdf

- Villardón, L. (Coord.) (2015). Competencias genéricas en educación superior. Metodologías específicas para su desarrollo. España: Narcea.

- Walker, M. y Nixon J. (2007). Reclaming universities from Runaway World. Londres: Open University Press.

- Yániz, C. y Villardón, L. (2006). Planificar desde competencias para promover el aprendizaje. Bilbao: Universidad de Deusto Cuadernos del ICE 12. 